\title{
AVALIAÇÃO DA ATIVIDADE ANTIULCEROGÊNICA DE Hamamelis virginiana L. E Sambucus australis Cham. \& Schltdl.
}

\section{EVALUATION OF ANTIULCER ACTIVITY OF Hamamelis virginiana L. AND Sambu- cus australis Cham. \& Schltdl.}

\author{
MARIA, M. D. ${ }^{1 *} ;$ DUDEK, D. N.2; TONETE, T. L. ${ }^{3}$ JORGE T. C. M.4; \\ SANCHES, A. C. C. ${ }^{5}$ \\ ${ }_{1,2,3}$ Aluna do Curso de Farmácia da Universidade Estadual do Oeste do Paraná, Unioeste, Cascavel, \\ PR, Brasil. ${ }^{4}$ Professora de Química Orgânica, Unioeste. ${ }^{5}$ Professora de Farmacologia, Unioeste. \\ "E-mail: marianadariomaria@ hotmail.com \\ REC: 02/2010 AC: 03/2010
}

\begin{abstract}
RESUMO
Úlcera é um tipo de inflamação que afeta um considerável número de pessoas no mundo, como muitas recorrem às plantas como um cuidado primário de saúde torna-se importante a realização de estudos sobre as mesmas. As plantas possuem componentes capazes de exercer ações farmacológicas. Já foram descritas para Hamamelis virginiana L. ação antiviral, antibacteriana, antiinflamatória, antioxidante. Enquanto para Sambucus australis Cham. \& Schltdl. são conhecidas ações antiinflamatórias e antioxidantes, tendo grande popularidade no tratamento sintomático de moléstias eruptivas, gripes e resfriados. $\mathrm{O}$ objetivo desse trabalho foi avaliar a atividade antiulcerogênica dos extratos brutos de $\mathrm{H}$. virginiana L. e S. australis Cham. \& Schltdl., frente ao modelo de úlcera induzido por etanol, em ratos. $O$ tratamento realizado foi de caráter preventivo, no qual se administrou cimetidina $100 \mathrm{mg} / \mathrm{Kg}$ (controle positivo), água destilada (controle negativo), H. virginiana L. $100 \mathrm{e}$ $200 \mathrm{mg} / \mathrm{Kg}$, S. australis Cham. \& Schltdl. 150 e $200 \mathrm{mg} / \mathrm{Kg}$, posteriormente a úlcera foi induzida. . Dos extratos avaliados apenas $H$. virginiana $L$. apresentou atividade antiulcerogênica significativa $(p<0,05), S$. australis Cham. \& Schltdl. não apresentou a mesma atividade, porém mostrou uma tendência à ação anti-edematogênico na dose de $150 \mathrm{mg} / \mathrm{Kg}$.
\end{abstract}

Palavras chave: antiulcerogênica, Hamamelis virginiana L., Sambucus australis Cham. \& Schltdl.

\begin{abstract}
Ulcer is a type of inflammation that affects a considerable number of people in the world, many of them use medicine plants as a primary health care, so it is important to conduct studies about plants. The plants have components capable to exercise pharmacological actions. It has already been described for Hamamelis virginiana L. antiviral, antibacterial, anti-inflammatory and antioxidant action. While Sambucus australis Cham. \& Schltdl. has known anti-inflammatory and antioxidant action, with great popularity in the symptomatic treatment of eruptive diseases, colds and flu. The object of this study was to evaluate the antiulcerogenic activity of extracts of $H$. virginiana L. e S. australis Cham. \& Schltdl., with the ulcer model induced by ethanol in rats. Preventive treatment was performed, which was administered cimetidine $100 \mathrm{mg} / \mathrm{kg}$ (positive control), distilled water (negative control), $\mathrm{H}$. virginiana L. 100 and $200 \mathrm{mg} / \mathrm{Kg}$, S. australis Cham. \& Schltdl. 150 e $200 \mathrm{mg} / \mathrm{Kg}$ and later the ulcer was induced. Only $H$. virginiana $L$. showed antiulcerogenic activity significant $(p<0,05)$. S. australis Cham. \& Schltdl. did not show the same activity, but showed a tendency an anti-edematogenic action at dose of $150 \mathrm{mg} / \mathrm{kg}$.
\end{abstract}

Keywords: evaluation, antiulcerogenic, Hamamelis virginiana L., Sambucus australis Cham. \& Schltdl. 


\section{INTRODUÇÃO}

A úlcera péptica era, até recentemente, considerada uma doença de etiologia desconhecida, de evolução em geral crônica, com surtos de ativação e acalmia (Coelho, 2001). É uma forma de inflamação caracterizada pela perda de tecido em uma superfície de pele ou das mucosas (Montenegro \& Fecchio, 1999). Diferencia-se das erosões pelo fato de estas não atingirem a submucosa e, portanto, não deixarem cicatrizes ao se curarem (Coelho, 2001). Tem como causa principal, um desequilíbrio entre a taxa de secreção do suco gástrico e do grau de proteção, que é desempenhado pela barreira mucosa e pela neutralização do ácido gástrico, ocasionado pelo suco duodenal. As úlceras gástricas e duodenais são doenças que afetam um considerável número de pessoas no mundo e são induzidas por fatores severos como: fumo, estresse, deficiências nutricionais, ingestão de drogas anti-inflamatórias não-esteróides, álcool e infecção por Helicobacter pylori (Guyton \& Hall, 2006).

Pesquisas desenvolvidas em Helsinque, na Finlândia, têm demonstrado que o H. pylori produz a enzima álcool-desidrogenase, $\mathrm{ADH}$, que oxida o etanol em acetaldeído, catabólito altamente tóxico e potencialmente capaz de lesar o epitélio gástrico, especialmente quando o $H$. pylori é incapaz de produzir a enzima aldeído-desidrogenase, que metabolizaria o acetaldeído produzido (Coelho, 2001).

São muitos os tratamentos para úlcera, mas, segundo Calixto (2000), devido à pobreza e à carência ao acesso à medicina moderna, $65-80 \%$ da população mundial, que vive em países subdesenvolvidos e em desenvolvimento, depende essencialmente das plantas como cuidado primário de saúde.

As plantas, além de seu uso na medicina popular com finalidades terapêuticas, têm contribuído, ao longo dos anos, para a obtenção de vários fármacos, até hoje amplamente utilizados na clínica. A avaliação do potencial terapêutico de plantas medicinais e de alguns de seus constituintes, tais como flavonóides, alcalóides, triterpenos, sesquiterpenos, taninos, lignanas, entre outros, têm sido objeto de incessantes estudos, nos quais já foram comprovadas as ações farmacológicas por meio de testes pré-clínicos com animais (Cechinel Filho \& Yunes, 1998). A atividade biológica e farmacológica dos taninos vem sendo amplamente estudada, verificando seus efeitos em enzimas, vírus, microrganismos e outras mais (Esaki, 1985).

Hamamelis virginiana pertence à família botânica Hamamelidaceae. Suas cascas e folhas contêm uma mistura complexa de taninos condensados e hidrolisáveis, e o hamamelitanino (2',5-di-O-galoilhamamelofuranose) é o principal constituinte da casca, mas outros taninos são conhecidos. Proantocianidinas diméricas também foram isoladas e identificadas (Santos \& Mello, 1999). Os taninos são responsáveis pela adstringência de muitos frutos e plantas em geral, através da sua complexação com proteínas, que é à base de algumas de suas propriedades biológicas, tais como controle de insetos, fungos e bactérias (Aerts et al, 1999). A fração de polifenol nas folhas é representada por uma mistura de galotaninos e proantocinidinas (Erdelmeier, 1996). Isolaram-se também, nas folhas, canferol, quercetina 3-O- -D-glucoronideo, cianidina, catequina, entre outros polifenóis (Sagareishvili et al., 1999).

Uma das ações relatadas de $H$. virginiana, é uma significante atividade in vitro contra o vírus Herpes simplex tipo 1 (HSV-1) e o vírus da imunodeficiência humana (HIV). De qualquer forma, a presença de polifenóis na molécula tem uma forte influ- 
ência tanto na capacidade antiviral como na polimerização. Consequentemente, o potencial de hidrolização dos taninos anti-HSV aumenta com o número de grupos polifenóis (Erdelmeier, 1996).

As folhas de $H$. virginiana foram avaliadas quanto à atividade antibacteriana, contra bactérias periodontais aeróbias e aeróbias facultativas (Porphyromonas gingivalis, Prevotella spp., Fusobacterium nucleatum, Capnocytophaga gingivalis, Veionella parvula, Eikenella corrodens, Peptostreptococcus micros e Actinomyces odontolyticus), e seu extrato metanólico apresentou uma atividade inibitória ( $\mathrm{MIC}<\mathrm{ou}=$ 2048mg/L), contra muitas dessas espécies testadas (lauk, 2003).

A ação anti-inflamatória também foi observada, no modelo de edema de pata em ratos e os resultados confirmaram a presença de substâncias responsáveis por esse efeito, mas mostrou-se ativa apenas contra a fase crônica (Duwiejua, 1994).

O hamamelitanino e o ácido gálico são potentes antioxidantes, mostrando ação superior ao ácido ascórbico. Em uma cultura de células, na qual o dano foi induzido por oxigênio ativo, tal como os ânions superóxidos, o hamamelitanino mostrou maior atividade que o ácido gálico contra tais radicais. Os dois compostos também apresentam atividade contra os radicais hidroxil, mas, em contrapartida, o ácido gálico mostra-se mais efetivo. O hamamelitanino apresenta ainda atividade antioxidante e sequestradora contra radicais orgânicos como DPPH (2,2-difenil-1, picril-hidrazila) (Masaki, 1994).

Além desses radicais, o peróxido de nitrito (ONOO-) representa um ânion citotóxico com fortes propriedades oxidantes voltado a vários constituintes celulares, incluindo sulfidrilas, lipídios, aminoácidos e nucleotídeos, podendo causar a morte celular, a peroxidação lipídica, a carcinogênese e o envelhecimento. $O$ extrato de $H$. virginiana exibiu atividade dose-dependente contra esse radical, sendo o hamamelitanino um componente com poderosos efeitos contra ONOO- (Choi et al, 2002). Foram também identificados compostos antioxidantes voláteis, através da cromatografia gasosa acoplada a espectrofotometria de massas (Silva et al., 2000).

O gênero Sambucus possui cerca de 25 espécies. Entre estas, destacam-se Sambucus canadensis L., Sambucus ebulus L., Sambucus nigra L. e Sambucus australis Cham. \& Schltdl., mencionadas como espécies medicinais em suas regiões de origem (Scopel et al., 2007). S. australis é nativo do Brasil, podendo ser encontrado nas regiões Nordeste e Sudeste até o Rio Grande do Sul, e S. nigra é uma espécie européia comercializada no país. Comparando-se $S$. australis com $S$. nigra, percebe-se que são discretas as diferenças entre estas espécies, que por vezes são consideradas como sinônimas (Nunes et al., 2007).

$S$. australis tem grande popularidade no tratamento sintomático de moléstias eruptivas, gripes e resfriados, por suas ações diaforética, anti-inflamatória e analgésica (Jorge et al., 1999).

Em screening realizado com amostra de $S$. australis, foi possível identificar a presença de flavonóides em grande quantidade, esteróis e triterpenos em menor quantidade (Alice et al., 1991, apud Scopel, 2005; Clemes et al., 2008). As flores dessa espécie apresentam flavonóides do tipo 3-O-monoglicosídeo de diidroflavonol, 3,7-O-diglicosídeo de flavonol, isoquercetina, rutina e quercetina, ácidos caféico e clorogênico (Alice et al., 1990, apud Scopel, 2005).

Scopel (2005) observou atividade anti-inflamatória com o ensaio de inibição do 
edema de pata em ratos, induzida por carragenina, para extratos aquosos e hidroetanólicos das flores de $S$. Nigra e $S$. Australis apresentando potência equivalente ao padrão indometacina. $\mathrm{O}$ mesmo autor analisou a atividade antioxidante in vitro por meio do método de doseamento colorimétrico do radical hidrato de 2,2-difenil-1, picril-hidrazila (DPPH) com os extratos aquosos e hidroetanólicos, verificando que o último apresentou atividade mais acentuada e maior percentual de rutina.

Alguns modelos estão disponíveis para testar substâncias e seus efeitos antiulcerogênicos (Goulart et al., 2005). Assim, o objetivo desse trabalho foi avaliar a atividade antiulcerogênica das drogas vegetais $H$. virginiana e Sambucus australis em um modelo animal, buscando-se comprovar sua etnofarmacologia.

\section{MATERIAL E MÉTODOS}

Animais: foram usados ratos Wistar machos, adultos, pesando entre $100 \mathrm{e}$ 250 gramas, provenientes do biotério central da Universidade Estadual do Oeste do Paraná, UNIOESTE, campus de Cascavel. Os animais foram levados ao biotério setorial do laboratório de Farmacologia para ambientação, submetidos a ciclo claro-escuro de 12 horas e mantidos à temperatura controlada, com livre acesso à água (Mizui \& Doteuchi, 1983) e à ração até 16h antes do experimento (Raphael \& Kuttan, 2003).

Drogas vegetais: folhas de $H$. virginiana (hamamelis), usadas na forma de extrato bruto liofilizado, adquirido em uma farmácia de Cascavel, Paraná (laudo do controle de qualidade em anexo); e planta total, excluindo-se as flores, de $S$. australis (sabugueiro) na forma de extrato bruto, oriundo do laboratório de Farmacognosia da Universidade Estadual do Oeste do Paraná e identificado pela Prof. ${ }^{a}$ Dr. ${ }^{a}$ Norma Catarina Bueno, cuja exsicata encontra-se depositada no herbário dessa universidade, sob no 2622.

Indução da úlcera: os ratos receberam etanol (96\%), por via oral (gavagem), como agente ulcerogênico (Del Soldato et al., 1984; Gürbüz et al., 2002), em doses de $1 \mathrm{~mL}$ por animal.

Tratamento Preventivo: os animais foram divididos em seis grupos, com cinco animais cada, e tratados por gavagem (v.o). O controle negativo recebeu $1 \mathrm{~mL}$ de água destilada. No segundo grupo, aplicou-se cimetidina (Teuto ${ }^{\circledR} 150 \mathrm{mg} / \mathrm{mL}$ ) na dose de $100 \mathrm{mg} / \mathrm{Kg}$ via intraperitoneal (i.p), como controle positivo. O terceiro e quarto grupo receberam extrato bruto de sabugueiro nas concentrações de $150 \mathrm{mg} / \mathrm{Kg}(8 \mathrm{mg} / \mathrm{mL})$ e $200 \mathrm{mg} / \mathrm{Kg}(12 \mathrm{mg} / \mathrm{mL})$, respectivamente. Ao quinto e sexto grupo administrou-se o extrato bruto liofilizado de hamamelis nas concentrações de $100 \mathrm{mg} / \mathrm{Kg}(6 \mathrm{mg} / \mathrm{mL})$ e $200 \mathrm{mg} / \mathrm{Kg}(12 \mathrm{mg} / \mathrm{mL})$. Ambos os extratos, em todas as concentrações, foram dissolvidos com água destilada.

Após 1 hora, administrou-se o agente ulcerogênico em todos os grupos. Posteriormente (60 minutos), os ratos foram sacrificados com gás carbônico. O estômago foi removido e aberto ao longo da pequena curvatura, da cárdia ao piloro, e lavados delicadamente com água destilada, para análise.

Análises macroscópicas: analisou-se coloração da mucosa, perda de pregas, edema, petéquias e úlceras.

As úlceras gástricas foram contadas e classificadas, segundo Bacchi et al. (1999) e Gonzalez et al. (2001), em 3 graus: 
- Úlcera de grau superficial (I): presença de pontos hemorrágicos superficiais com área de até $1 \mathrm{~mm}^{2}$;

- Úlcera grau moderado (II): presença de lesões hemorrágicas na submucosa com pequenas erosões com áreas entre 1 e $3 \mathrm{~mm}^{2}$;

- Úlcera grau severo (III): presença de lesões erosivas hemorrágicas com área maior que $3 \mathrm{~mm}^{2}$.

O índice de úlcera (IU) foi calculado para cada animal, segundo Goulart et al. (2005) e Gonzalez et al. (2001):

$\mathrm{IU}=(\mathrm{n}$ úlcera $\mathrm{I})+2(\mathrm{n}$ úlcera $\mathrm{II})+3(\mathrm{n}$ úlcera $\mathrm{III})$, em que $n$ representa o número de úlceras.

Análise Estatística: os resultados dos índices de úlceras foram analisados pela média \pm erro padrão da média para cada grupo. Os grupos foram avaliados entre si usando o teste ANOVA, acompanhado pelo Dunnett's. As diferenças foram consideradas estatisticamente significantes quando $p \leq 0,05$.

\section{RESULTADOS E DISCUSSÃO}

Por meio da avaliação macroscópica foi possível observar que, com relação a edema (Tabela 1), 25\% dos animais tratados com hamamelis $100 \mathrm{mg} / \mathrm{kg}$, apresentaram alteração e, nesse mesmo grupo, $75 \%$ dos animais apresentaram a presença de pregas. Isso mostra que são sinais inversamente proporcionais ao edema. A coloração esteve alterada em $20 \%$ dos animais tratados com sabugueiro $150 \mathrm{mg} / \mathrm{kg}$ e em $25 \%$ dos animais tratados com hamamelis $100 \mathrm{mg} / \mathrm{kg}$. Sabe-se que esses são sinais devido à injúria no tecido causado pelo álcool (Gaskil et al., 1982, apud Goulart et al., 2005), que aumenta a permeabilidade vascular e aumenta o fluxo sanguíneo na região inflamada (Smyth, Burke \& Fitzgerald, 2007). Tais alterações são características de inflamação, devido à agressão provocada por esse agente.

Tabela 1 - Comparação de alguns parâmetros analisados: edema, pregas e coloração.

\begin{tabular}{cccc}
\hline & $\begin{array}{c}\text { Presença de } \\
\text { Edema (\%) }\end{array}$ & $\begin{array}{c}\text { Presença de } \\
\text { Pregas (\%) }\end{array}$ & $\begin{array}{c}\text { Alteração na } \\
\text { Coloração (\%) }\end{array}$ \\
\hline Controle Negativo & 75 & 25 & 50 \\
Controle Positivo & 50 & 75 & 25 \\
Hamamelis 100mg/Kg & 25 & 75 & 25 \\
Hamamelis 200mg/Kg & 20 & 60 & 60 \\
Sabugueiro 150mg/Kg & 20 & 20 & 20 \\
Sabugueiro 200mg/Kg & 40 & 40 & 60 \\
\hline
\end{tabular}

Com relação à presença de petéquias, não houve alterações estatisticamente significativas.

Quanto ao índice de úlcera, foi possível observar que os animais tratados com cimetidina $100 \mathrm{mg} / \mathrm{kg}$ tiveram redução nesse parâmetro, quando comparados ao grupo controle negativo (água destilada), o que mostra a validade dessa metodologia. As 
diferentes concentrações de hamamelis mostraram resultados estatisticamente significativos para o índice de úlcera $(p=0,0281)$, quando comparados ao controle negativo. Entretanto, os extratos de sabugueiro não foram efetivos, $p>0,05$ (Figura 1).

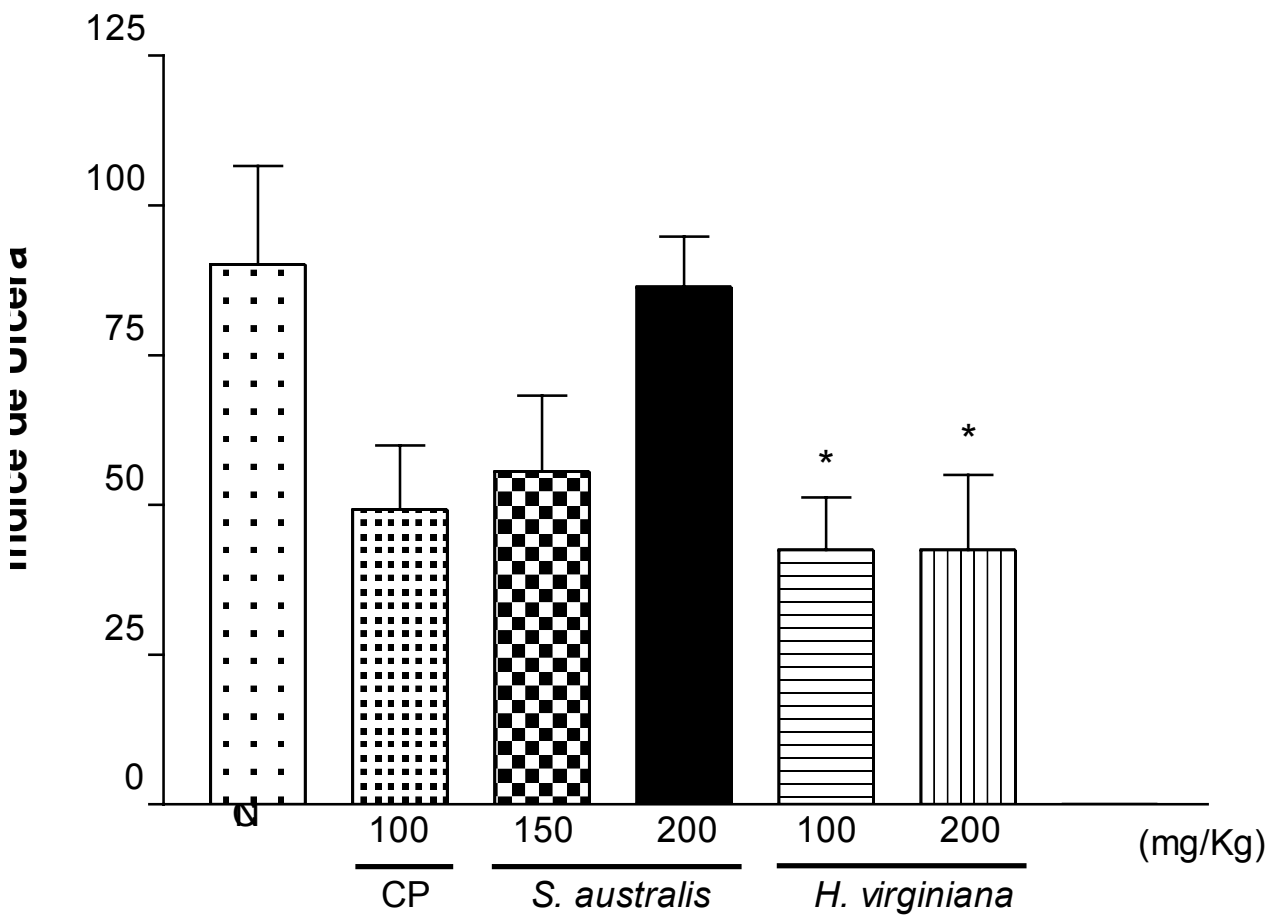

Figura 1 - Ação antiulcerogênica dos extratos de Sambucus australis Cham. \& Schlcht. e Hamamelis virginiana L. comparados com o controle negativo (CN, água destilada). ANOVA/ Dunnett's, * $p=0,0281$.

As células hepáticas convertem o etanol em um composto de maior toxicidade, o etanaldeído, que reduz os níveis de glutationa no fígado, ocasionando peroxidação lipídica e desencadeando a produção de radicais livres (Raphael \& Kuttan, 2003), tornando-se importante a presença de compostos antioxidantes, como taninos e flavonóides, nas drogas vegetais. Vennat et al., (1992); Masaki et al., (1994); Haslam (1996); Sagareishvili et al., (1999); Silva et al., (2000); Choi et al., (2002); Tourino et al., (2008); relataram a existência de compostos responsáveis por essa atividade em $H$. virginiana. Já foi também demonstrado que os taninos estão associados com atividade antiulcerogênica em outras plantas (Esaki et al., 1985) e que auxiliam na cicatrização, devido à sua capacidade de complexação com proteínas, formando uma película sobre a região danificada (Lopes et al., 2005).

A comparação da mucosa gástrica, após o tratamento preventivo com as drogas vegetais, pode ser realizada por meio da figura 2. 


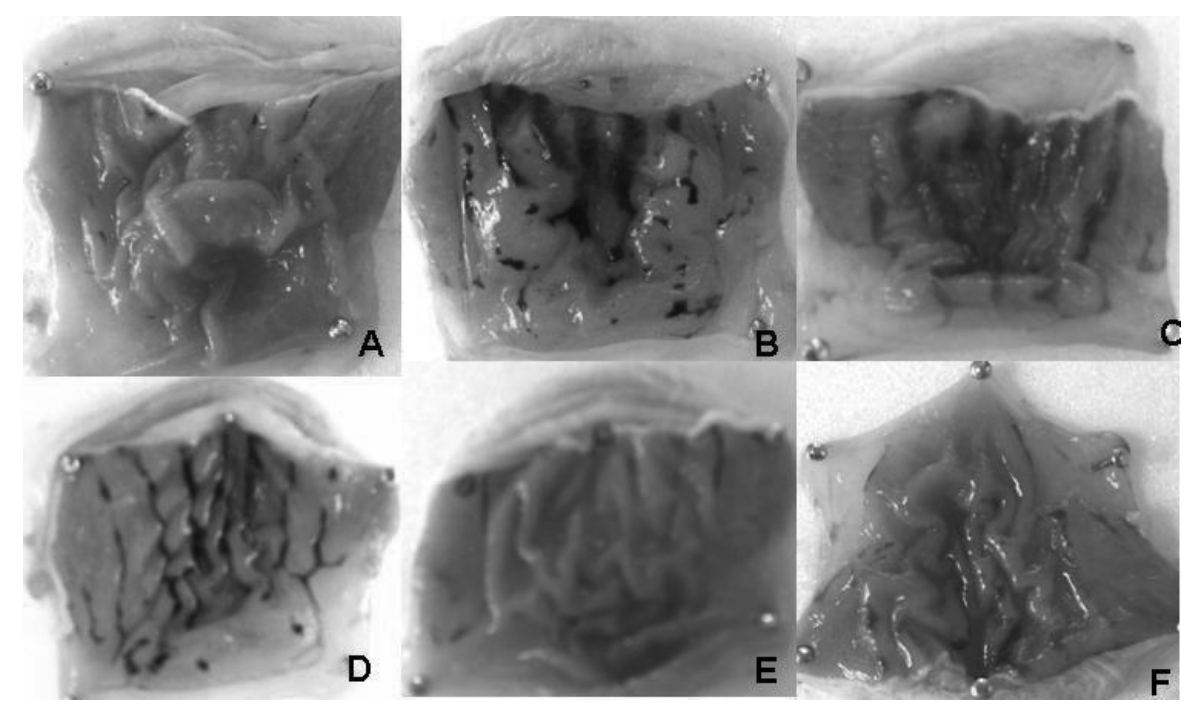

Figura 2 - Comparação da mucosa gástrica após tratamento preventivo entre: controle positivo (A, cimetidina $100 \mathrm{mg} / \mathrm{Kg}$ ), controle negativo (B, água destilada), S. australis Cham. \& Schlcht. 150 e $200 \mathrm{mg} / \mathrm{Kg}$ (C e D, respectivamente), H. virginiana L. 100 e $200 \mathrm{mg} / \mathrm{Kg}$ (E e F, respectivamente).

Takahashi et al. (2002) encontrou, em extrato isolado de pelo menos uma planta selecionada de vários gêneros, incluindo $H$. virginiana, compostos antagonistas do receptor histamínico $\mathrm{H} 2$. Assim, o extrato em questão é útil para essa atividade farmacológica, podendo inibir a secreção gástrica, prevenindo úlceras pépticas.

Com relação à espécie de sabugueiro avaliada, não foi encontrada atividade antiulcerogênica, mesmo essa droga apresentando flavonóides que são substâncias com ação antioxidante, sequestradora de radicais livres e quelante de oxigênio (Scopel, 2005; Harborne \& Williams, 2000).

Ainda que haja estudos da presença de polifenóis nas folhas de $S$. australis (Clemes et al., 2008), a literatura mostra, principalmente, a existência de compostos como flavonóides, isoquercetina, rutina, entre outros, nas flores dessa planta (Alice et al., 1990, apud Scopel, 2005), o que poderia justificar a ausência de ação antiulcerogênica, já que o extrato foi produzido com a planta inteira, porém excluindo-se as flores.

Entretanto, outra espécie desse mesmo gênero, Sambucus ebulus, demonstrou significante atividade antiulcerogênica quando administrado oralmente seu extrato aquoso em ratos (Yesilada et al., 1992).

Adicionalmente, fatores protetores da mucosa gástrica, bicarbonato e muco, são produzidos graças à ação das prostaglandinas (PGEs, $\mathrm{PGI}_{2}$ ), compostos que também inibem a secreção de ácido e aumentam o fluxo sanguíneo.

Enquanto esses compostos e a prostaciclina são citoprotetores, o tromboxano $A_{2}\left(T X A_{2}\right)$ é ulcerogênico no cão. Já os leucotrienos podem desempenhar um papel na inflamação e na lesão tissular. Portanto, substâncias que inibem as enzimas ciclo-oxigenases (COX) e lipoxigenases (LOX) têm importante efeito anti-inflamatório (Silva, 2006). Contudo, a inibição de COX-1, expressada constitutivamente, provoca redução da proteção gástrica (Carvalho, 2006). Isso pode ter ocorrido no presente estudo, visto que segundo Harborne \& Williams (2000), os flavonóides podem interferir na rota das enzimas COX e/ou 5-LOX do metabolismo do ácido araquidônico e estes comp- 
ostos são encontrados nas duas plantas analisadas. Hartisch (1997) demonstrou que o hamamelitanino e a proantocinidina com radical galoil de $H$. virginiana são potentes inibidores da 5-LOX.

\section{CONLUSÃO}

Dos extratos testados, apenas $H$. virginiana demonstrou atividade antiulcerogênica, quando comparado ao controle negativo, e uma tendência à atividade superior à cimetidina. Provavelmente isso se deve ao fato de haver vários componentes com atividades biológicas em sua composição, os quais podem agir sinergicamente. Nesse estudo, não se observou um possível efeito dose-dependente, já que as concentrações de 100 e $200 \mathrm{mg} / \mathrm{Kg}$ obtiveram índices de úlceras muito semelhantes. Apenas o edema é favorável à concentração maior, possivelmente por uma ação mecânica protetora do extrato na mucosa gástrica. Entretanto, logicamente, esse único fator não justificaria o uso da droga nessa concentração, principalmente porque, aumentando a dose, pode-se aumentar a toxicidade.

O extrato de $S$. australis não apresentou atividade antiulcerogênica mesmo possuindo polifenóis, possivelmente por não estarem na concentração adequada para atingir tal ação. Nessa droga também não se observou possível efeito dose-dependente, tendo em vista que o índice de úlcera dos ratos que receberam a concentração de $200 \mathrm{mg} / \mathrm{Kg}$ não foi superior à concentração de $150 \mathrm{mg} / \mathrm{Kg}$, e os parâmetros edema e coloração também foram piores com relação à menor concentração. Entretanto, na dose de $150 \mathrm{mg} / \mathrm{Kg}$, mostrou uma tendência à ação antiedematogênica e manteve a coloração da mucosa gástrica normal na maioria dos estômagos analisados.

Sendo assim, sugerimos trabalhos futuros que avaliem a toxicidade das duas plantas e a repetição desse próprio teste, principalmente no caso de $S$. australis, usando extrato que inclua as flores em sua preparação, já que as mesmas não estavam presentes no extrato em estudo.

\section{AGRADECIMENTOS}

Agradecemos ao Biotério Central, ao Laboratório de Farmacologia e ao Laboratório de Farmacognosia da Universidade Estadual do Oeste do Paraná.

\section{REFERÊNCIAS}

AERTS, T. J.; BARRY, T. N.; MCNABB, W. C. Polyphenols and agriculture: beneficial effects of proanthocyanidins in forages. Agriculture, Ecosystems and Environment. 1999,75, 1-12.

ALICE, C. B.; SIQUEIRA, N. C. S.; HALBIG, J. C.; MENTZ, L. A.; SILVA, G. A. A. B.; GOETTEMS, E. I. Diagnose comparativa de compostos polifenólicos de Sambucusnigra L. e Sambucus australis Cham. \& Schlecht. Revista Brasileira de Farmácia. 1990, 71(4), 88-90. apud SCOPEL, M.; Análise Botânica, Química e Biológica Comparativa entre Flores das Espécies Sambucus nigra L. e Sambucus australis Cham. 
\& Schltdl. e Avaliação Preliminar da Estabilidade. Tese de Mestrado, Universidade Federal do Rio Grande do Sul, 2005.

ALICE, C. B.; VARGAS, V. M. F.; SILVA, G. A. B.; SIQUEIRA, N. C. S.; SCHAPOVAL, E. E. S.; GLEYDE, J.; HENRIQUES, J. A. P.; HENRIQUES, A. T. Screening of plants used in south Brazilian folk medicine. Journal of Ethnopharmacology. 1991, 35(2), 165-171. apud SCOPEL, M.; Análise Botânica, Química e Biológica Comparativa entre Flores das Espécies Sambucus nigra L. e Sambucus australis Cham. \& Schltdl. e Avaliação Preliminar da Estabilidade. Tese de Mestrado, Universidade Federal do Rio Grande do Sul, 2005.

BACCHI, E.M.; SERTIÉ, J.A.A.A.; SUFFREDINI, I.B. Antiulcer action of Microgramma squamulosa (Kaulf.) Sota. Journal of Ethnopharmacology. 1999, 65(3), 217-223.

CALIXTO, J. B.; Eficacy, safety, quality control, marketing and regulatory guidelines for herbal medicines (phytotherapeutic agents). Brazilian Journal of Medical and Biological Ressearch. 2000, 33(2), 179-189.

CARVALHO, W. A. Antiinflamatórios não-esteróides, analgésicos, Antipiréticos e drogas utilizadas no tratamento da gota In Farmacologia, Silva, P. Ed.: Guanabara Koogan (7ed.). Rio de Janeiro, 2006, 443.

CECHINEL FILHO, V.; YUNES, R. A. Estratégias para a obtenção de compostos farmacologicamente ativos a partir de plantas medicinais: conceitos sobre modificação estrutural para otimização da atividade. Quím. Nova. 1998, 21(1), 99-105.

CHOI, H. R.; CHOI, J. S.; HAN, Y. N.; BAE, S. J.; CHUNG, H. Y. Peroxynitrite scavenging activity of herb extracts. Phytotherapy Research. 2002, 16(2), 364-367.

CLEMES, S. M.; ZENI, A. L. B.; KRETZSCHMAR, M. Chemical evaluation of leaves from medicinal native plants used in Itajai Montainrange National Park and surroundings. Revista Brasileira de Farmácia. 2008, 89(1), 10-12.

COELHO, L. G. V. Úlcera Péptica Gastroduodenal In Gastroenterologia Essencial, Dani, R. Ed.: Guanabara Koogan (2ed.). Rio de Janeiro, 2001; 149-153.

DEL SODATO, P.; FOSCHI, D.; VARIN, L.; DANIOTTI, S. Comparison of the gastric cytoprotective properties of atropine, ranitidine and PGE2 in rats. Journal European of Pharmacology, 1984, 106(1), 53-58.

DUWIEJUA, M.; ZEITLIN, I. J.; WARWEMAN, P. G.; GRAY, A. I. Anti-inflamatory activity of Polygonum bistorta, Guaiacum officinale and Hamamelis virginiana in rats. Journal of Pharmacy and Pharmacology. 1994, 46(4), 286-290.

ERDELMEIER, C. A. J.; CINATL, J., JR.; RABENAU, H.; DOERR, H. W.; BIBER, A.; $\mathrm{KOCH}$, E. Antiviral and anti-inflamatory activities of Hamamelis virginiana. Planta Medica. 1996, 62(3), 241-245.

ESAKI, N.; KATO, M.; TAKIZAWA, N.; MORIMOTO, S.; NOKARA, G. I.; NISHIOKA, I. 
Pharmacological studies on Lindera umbellatae Ramus, IV. Effects of condensed tannin related compounds on peptic activity and stress-induced gastric lesions in mice. Planta Medica. 1985, 46, 34-38.

GASKIL, D. L.; SCRINCK, K. L.; LEVINE, V. A. Effect of prostacyclin on mucosal blood flow. Surgery.1982, 92, 220-224. apud GOULART, Y. C. F.; SELA, V. R.; OBICI, S.; MARTINS, J. V. C.; OTOBONE, F.; CORTEZ, D. A.; AUDI, E. A. Evaluation of gastric anti-ulcer activity in a hydroethanolic extract from Kielmeyera coriacea. Brazilian Archives of Biology and Technology. 2005, 48(1), 211-216.

GONZALEZ, F. G.; PORTELA, T. Y.; STIPP, E. J.; DI STASI, L. C. Antiulcerogenic and analgesic effects of Maytenus aquifolium, Sorocea bomplandii and Zolernia ilicifloia. Journal of Ethnopharmacology. 2001, 77(1), 41-47.

GOULART, Y. C. F.; SELA, V. R.; OBICI, S.; MARTINS, J. V. C.; OTOBONE, F.; CORTEZ, D. A.; AUDI, E. A. Evaluation of gastric anti-ulcer activity in a hydroethanolic extract from Kielmeyera coriacea. Brazilian Archives of Biology and Technology. 2005, 48(1), 211-216.

GÜRBÜZ, I.; ÜSTÜN, O.; YESILADA, E.; SEZIK, E.; AKYÜREK, N. In vivo gastroprotective effects of five Turkish folk remedies against ethanol-induced lesions. Journal of Ethnopharmacology. 2002, 83, 241-244.

GUYTON, A. C.; HALL, J. E. Fisiologia dos Distúrbios Gastrointestinais In Tratado de Fisiologia Médica. Ed.: Elsevier (11ed.). Rio de Janeiro, 2006; 820-821.

HARBORNE, J. B.; WILLIAMS, C. A. Advances in flavonoid research since 1992. Phytochemistry. 2000, 55(6), 481-504.

HARTISCH, C.; KOLODZEIJ, H.; VON BRUCHHAUSEN, F. Dual inhibitory activities of tannins from Hamamelis virginiana and related on 5-lipoxygenase and lyso-PAF. Acetyl-CoA acetyltransferase. Planta Medica. 1997, 63(2), 106-110.

HASLAM, E. Natural Polyphenols (Vegetable Tannins) as Drugs: Possible Modes of Action. J. Nat. Prod. 1996, 59 (2), 205-215.

IAUK, L.; LO BLUE, A. M.; MILAZZO, I.; RAPISARDA, A.; BLANDINO, G. Antibacterial activity of medical plant extracts agains periodontopathic bacteria. Phytotherapy Research. 2003, 17(6), 599-604.

JORGE, L. I. F.; GRACIANO, R. A. S.; PRADO, S. P. T.; PEREIRA, U. Identificaçäo histológica de Sambucus australis Cham. \& Schlecht. (Sabugueiro). Rev. Ciên. Farm. 1999, 20(1), 117-123.

LOPES, G. C.; SANCHES, C. C. A.; NAKAMURA, C. V.; DIAS FILHO, B. P.; HERNANDES, L.; MELLO, J. C. P. Influence of extracts of Stryphnodendron polyphyllum Mart. and Stryphnodendron obovatum Benth. on the cicatrisation of cutaneous wounds in rats. Journal of Ethnopharmacology. 2005, 99(2), 265-272. 
MASAKI, H.; ATSUMI, T.; SAKURAI, H. Hamamelitannin as a new potent active oxygen scavenger. Phytochemistry. 1994, 37(2), 337-343.

MIZUI, T.; DOTEUCHI, M.; Effect of polyamines on acidified ethanol-induced gastric lesions in rats. Journal of Pharmacology. 1983, 33, 939-945.

MONTENEGRO M. R.; FECCHIO, D. Inflamação: Conceitos Gerais e Inflamação Aguda In Patologia: Processos Gerais, M. R. Montenegro, M. Franco. Ed. Atheneu (4ed.). São Paulo, 1999; 127.

NUNES, E.; SCOPEL, M.; VIGNOLI-SILVA, M.; VENDRUSCOLO, G. S.; HENRIQUES, A. T.; MENTZ, L. A. Caracterização farmacobotânica das espécies de Sambucus (Caprifoliaceae) utilizadas como medicinais no Brasil. Parte II. Sambucus australis Cham. \& Schltdl. Rev. Brasileira de Farmacognosia. 2007, 17(3), 414-425.

RAPHAEL, K.R.; KUTTAN, R. Inhibition of experimental gastric lesion and inflammation by Phyllanthus amarus extract. Journal of Ethnopharmacology. 2003, 87, 193197.

SAGAREISHVILI, T. G.; YAROSH, E. A.; KEMERTELIDZE, E. P. Phenolic compounds from leaves of Hamamelis virginiana. Chemistry of Natural Compounds. 1999, 35(5), 585

SANTOS, S. C.; MELLO, J. C. P. Taninos In Farmacognosia: da planta ao medicamento, C. M. O. Simões, E. P. Schenkel, G. Gosmann, J. C. P. Mello, L. A. Mentz, P. R. Petrovick (orgs.). Ed.: Editora da Universidade-Editora da UFSC (1ed.). Porto Alegre-Florianópolis, 1999; 536.

SCOPEL, M.; Análise Botânica, Química e Biológica Comparativa entre Flores das Espécies Sambucus nigra L. e Sambucus australis Cham. \& Schltdl. e Avaliação Preliminar da Estabilidade. Tese de Mestrado, Universidade Federal do Rio Grande do Sul, 2005.

SCOPEL, M.; NUNES, E.; VIGNOLI-SILVA, M.; VENDRUSCOLO, G. S.; HENRIQUES, A. T.; MENTZ, L. Caracterização farmacobotânica das espécies de Sambucus (Caprifoliaceae) utilizadas como medicinais no Brasil. Parte I. Sambucus nigra L. Rev. Brasileira de Farmacognosia. 2007, 17(2), 249-261.

SILVA, A. P.; ROCHA, R.; SILVA, C. M. L.; MIRA, L.; DUARTE, M. F.; FLORENCIO, M. $\mathrm{H}$. Antioxidants in medicinal plant extracts. A research study of the antioxidant capacity of Crataegus, Hamamelis abd Hydrastis. Phytotherapy Research. 2000; 14(8), 612-616.

SILVA, P. Autacóides In Farmacologia, Silva, P. Ed.: Guanabara Koogan (7ed.). Rio de Janeiro, 2006, 574-575.

SMYTH, E. M.; BURKE, A.; FITZGERALD, G. A. Autacóides derivados de lipídios: eicosanóides e fator de ativação das plaquetas In As Bases Farmacológicas da 
Terapêutica, Goodman, L. S.; Gilmam, A. Ed.: McGraw-Hill (11ed.). Rio de Janeiro, 2007, 596.

TAKAHASHI, Y.; YAMAGUCHI, S.; INOUE, S.; YOSHIDA, M. Histamine H2 receptor antagonistic agent and skin care preparation. J.P. Patent 2002029979 A 20020129 , 2002.

TOURINO, S.; LIZARRGA, D.; CARRERAS, A.; LORENZO, S.; UGARTONDO, V.; MITJANS, M.; VINARDELL, M. P.; LUIS, J.; CASCANTE, M.; TORRES, J. L. Highly galloylated tannin fractions from witch hazel (Hamamelis virginiana) bark: electron transfer capacity, in vitro antioxidant activity, and effects on skin-related cells. Chemical research in toxicology. 2008, 21(3), 696-704.

VENNAT, B.; GROSS, D.; POURRAT, H. Hamamelis virginiana: identification and assay of proanthocyanidins, phenolic acids and flavonoids in leaf extracts. Pharmaceutica Acta Helvetiae. 1992, 67(1), 11-14.

YESILADA, E.; SEZIK, E.; FUJITA, T.; TANAKA, S.; TABATA, M. Screening of some Turkish medicinal plants for their antiulcerogenic activities. Phytotherapy Research. 1992, 7(3), 263-265. 